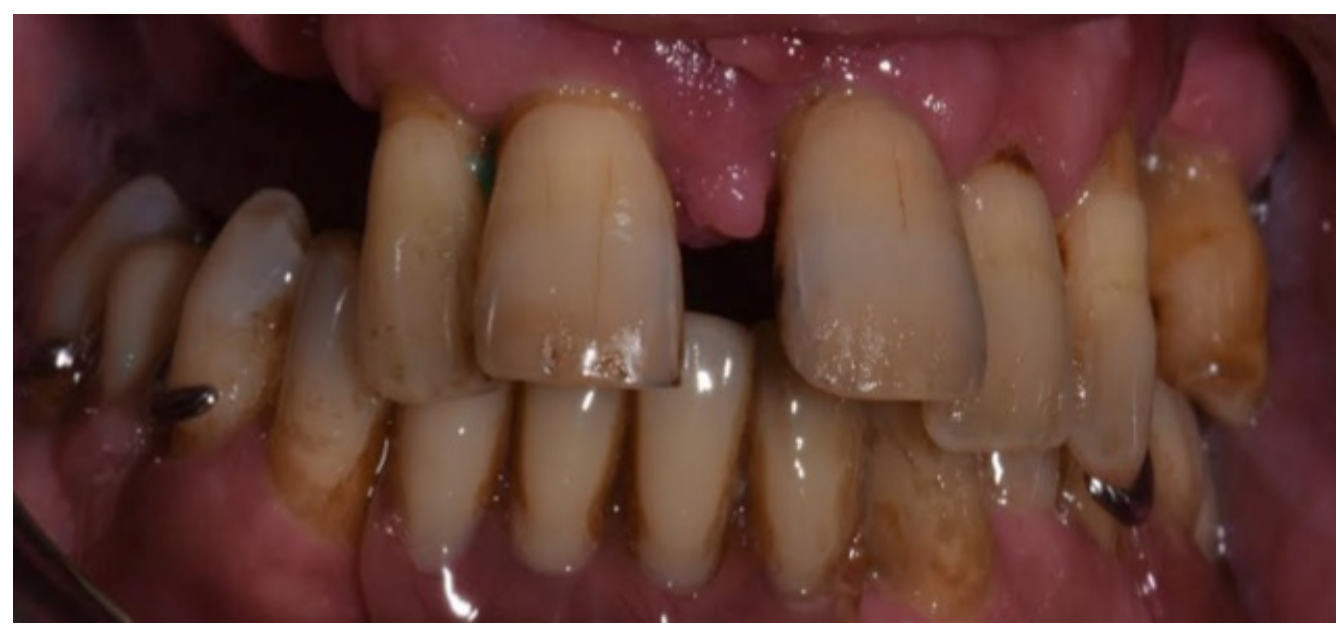

\title{
A new exciting hypothesis: direct correlation between periodontitis and clinical evolution of COVID-19 patients. Preprint.
}

\author{
Giuseppina Campisi ${ }^{1}$, Maria Eleonora Bizzoca², Lorenzo Lo Muzio² \\ 1 University of Palermo \\ 2 University of Foggia
}

Funding: The author(s) received no specific funding for this work.

Potential competing interests: The author(s) declared that no potential competing interests exist.

\section{Abstract}

The aim of the present opinion article is to hypothesize a possible correlation between clinical evolution of COVID-19 and a chronic inflammatory disease such as periodontitis.

Reviews have been performed about the correlations between COVID-19 and IL-6, COVID-19 and diabetes, diabetes and periodontitis.

Several studies discussed the role played by IL- 6 in the pathogenetic mechanisms of COVID-19. A recent study found that COVID-19 adult patients with diabetes, without other comorbidities, were at greater risk of numerous diseases COVID related, such as severe pneumonia and excessive uncontrolled inflammatory responses. Besides, serum levels of inflammation-related biomarkers, such as IL-6, were found to be 
higher in diabetic patients than healthy subjects, suggesting that patients with diabetes are more susceptible to the inflammatory storm that leads to rapid health deterioration of COVID-19 patients.

Our hypothesis origins from strict relationship, established, between periodontitis, as being able to induce production of IL-6, and diabetes in adult patients. Hence, by evaluating the associations previously described (COVID-19 and IL-6, COVID-19 and diabetes, IL-6 and diabetes) one wonders if, at least in diabetic patients, there could be a role for periodontitis, as a systemic risk factor, in the worst COVID-19 evolution. Periodontitis and oral health status can be very important in the evaluation of COVID19 behavior and the clinicians must examine the oral health status in these patients.

post scriptum. Authors equally contributed

\section{Main text}

Coronavirus disease 2019 (COVID-19), due to a SARS-CoV-2, is the most emergent health worldwide problem. While most cases result in mild symptoms, some progress to severe pneumonia and multi-org an failure with the death of the patient according to the age and the presence of other health problems. Data from Wuhan, China, note that the majority ( $51 \%$ - $66.7 \%)$ of affected patients have been male.

Recent literature pointed out the possible role of IL- 6 in the pathogenetic mechanisms of COVID-191: when SARS-CoV-2 infect the respiratory tract, it induce the release of proinflammatory cytokines, including interleukin (IL)-1 beta and IL-61. One of the mechanisms can make the coronavirus lethal is the induction of interstitial pneumonia linked to an over-production of IL-61. Based on this principle, several researchers started the use of an anti-arthritis drug, tocilizumab, an immunosuppressant able to block interleukin-6 production 1. The greatest experience with this drug comes precisely from the treatment of tumors with immunotherapy: when the immune system is stimulated to fight the tumor, there is a side effect that is respiratory distress, very similar to that by Covid-19, and tocilizumab reduces the excessive and counterproductive action of the immune system which causes inflammation of the pulmonary alveoli 1.

A recent study pointed out that COVID-19 patients without other comorbidities but with diabetes were at higher risk of severe pneumonia, release of tissue injury-related enzymes, excessive uncontrolled inflammation responses and hypercoagulable state associated with dysregulation of glucose metabolism2. Furthermore, serum levels of inflammation-related biomarkers such as IL-6, C-reactive protein, serum ferritin and coagulation index, D-dimer, were significantly higher $(P<.01)$ in diabetic patients compared with those without, suggesting that patients with diabetes are more 
susceptible to an inflammatory storm eventually leading to rapid deterioration of COVID192. Huang et al. in a systematic review and meta-analysis showed that diabetes was associated with mortality, severe COVID-19 and ARDS in SARS-COV-2 positive patients 3. There is evidence of increased incidence and severity of COVID-19 in patients with diabetes 4, and conversely COVID-19 could have effect on the pathophysiology of diabetes4.

In last years, there was a particular interest in confirming associations between periodontitis and systemic disease. Periodontitis is considered a chronic multifactorial inflammatory disease due to presence of dental plaque. This condition causes the progressive destruction of the tooth-supporting apparatus. Periodontitis is a worldwide diffuse disease, and in China it was estimated that its prevalence, for the severe variant, is $1.9 \%$ and that people with diabetes are 2.4 times more likely to have this variant; periodontitis has a documented higher prevalence in adult men $(\sim 57 \%)$ as compared to women ( 39\%) signifying a possible sex/gender involvement in the disease pathogenesis 5. Indeed, periodontal pathogens and their products, including inflammatory mediators such as IL-6, could enter the bloodstream, and chronic periodontitis has been found to be a risk factor for diabetes mellitus, and still being studied for cardiovascular disease, respiratory disease, rheumatoid arthritis and others. The systemic inflammatory burden of periodontitis is well-documented also thanks to the evidence of high sensitivity Creactive protein (hSCRP).

Very interestingly, the strict relationship among periodontitis, diabetes and IL-6 was demonstrated by a recent in vivo experimental study: coexistent diabetes and periodontitis significantly reduced leptin and IL-18 and significantly increased IL- 6 than controls, concluding that periodontitis is able to induce changes indicative of systemic inflammation, and it is considered the sixth major complication of individuals with diabetes 6 . There are several biological mechanisms that explain the connection between periodontitis and diabetes. For example, chronic activation of the immune system with increased levels of circulating leukocytes and pro-inflammatory markers has been reported in people with inadequately controlled diabetes 6 . Furthermore, hyperglycemia seems to alter systemic and ging ival microvasculature, leading to increased inflammation of the periodontal tissues. Data from epidemiology studies as well as animal studies suggest that the presence of one condition tends to increase the risk and severity of the other. It is widely accepted that diabetes mellitus is a risk factor for increased prevalence and severity of periodontitis. Moreover, healthy individuals with periodontitis exhibit a poor glycaemic control and a higher risk of developing diabetes 7; individuals affected by diabetes show a deterioration of glycaemic control if also affected by periodontitis and 
significantly higher prevalence of diabetes-related complications 7.

The analysis of the robust and already proven associations between periodontitis and IL6 and COVID-19 and IL-6, at least in diabetic patients, invite the scientific community to make stimulating hypotheses, as the following:

a) could periodontitis (initially a local disease followed by a systemic cascade of events) be a co-risk factor involved in the more agg ressive clinical manifestation of COVID-19 in some, at least diabetic, SARS-CoV-2 positive -especially men- patients?

b) could periodontitis be able to induce such a chronic inflammatory status able to sustain stably the release of cytokines as IL-6, the same mediator as the adverse events in COVID-19?

c) could be beneficial to clinically evaluate and monitor the periodontal status of diabetic SARS-COV-2 positive patients over time, in order to check IL-6 production?

Robust studies, based on the evidence, are suggested to elucidate these potential relationships and their pathophysiologic and clinical impact on the worldwide community. Authors equally contributed

\section{References}

1. Zhang C, Wu Z, LiJW, Zhao H, Wang GQ. The cytokine release syndrome (CRS) of severe COVID-19 and Interleukin- 6 receptor (IL-6R) antagonist Tocilizumab may be the key to reduce the mortality. Int J Antimicrob Agents. 2020:105954.

2. Guo W, Li M, Dong Y, et al. Diabetes is a risk factor for the progression and prognosis of COVID-19. Diabetes Metab Res Rev. 2020:e3319.

3. Huang I, Lim MA, Pranata R. Diabetes mellitus is associated with increased mortality and severity of disease in COVID-19 pneumonia - A systematic review, metaanalysis, and meta-regression. Diabetes Metab Syndr. 2020;14(4):395-403.

4. Singh AK, Gupta R, Ghosh A, Misra A. Diabetes in COVID-19: Prevalence, pathophysiology, prognosis and practical considerations. Diabetes Metab Syndr. 2020;14(4):303-310.

5. Ioannidou E. The Sex and Gender Intersection in Chronic Periodontitis. Front Public Health. 2017;5:189.

6. Corbella S, Francetti L, T aschieri S, De Siena F, Fabbro MD. Effect of periodontal treatment on glycemic control of patients with diabetes: A systematic review and metaanalysis. J Diabetes Investig. 2013;4(5):502-509.

7. Graziani F, Gennai S, Solini A, Petrini M. A systematic review and meta-analysis of epidemiologic observational evidence on the effect of periodontitis on diabetes An update of the EFP-AAP review. J Clin Periodontol. 2018;45(2):167-187. 
